\title{
Le site de Cassagna 2 (Blagnac, Haute-Garonne), une petite série problématique :
} Yaramila TCHÉRÉMISSINOFF rappel des faits en forme d'introduction

\begin{abstract}
Résumé
Le site de Cassagna 2 a fait l'objet d'un article détaillé il y a environ deux ans dans ce même bulletin. L'attribution à un faciès campaniforme de la petite série présentée avait alors fait l'objet de vives critiques, notamment de la part du comité de lecture, en regard du déficit d'éléments typologiques et du profil taphonomique du gisement. Cependant, les questions soustendues par cette réflexion se sont finalement avérées utiles au regard de cette productive table ronde. En effet, la définition de ces faciès «innommables», c'est-à-dire comportant des éléments mixtes situés entre le XXIII et le XXe siècle avant notre ère, reste compliquée, ne serait-ce que parce qu'ils sont le plus souvent escamotés sous l'appellation générique de Chalcolithique/Bronze ancien, alors qu'ils semblent plutôt déterminants en vue de la qualification même du Bronze ancien I dans de nombreuses régions.

Abstract

The site of Cassagna 2 was the subject of a detailed paper two years ago in this bulletin. The attribution then to a «Bell Beaker» facies of the small series presented was the object of strong criticism, notably from the editorial board, due to the lack of typological elements and the taphonomic profile of the deposit. However, the questions underlying this reflection finally proved useful with respect to this productive meeting. The definition of these «unnameable» facies, i.e. containing «mixed» elements situated between the $23 \mathrm{rd}$ and 20 th centuries B.C., remains complicated, because they are mostly eluded under the generic term «Chalcolithic/Early Bronze Age», although they seem rather decisive in the qualification of Early Bronze I in many regions.
\end{abstract}

\section{LES DONNÉES}

Le site de Cassagna 2 a fait l'objet d'un article détaillé il y a environ deux ans dans ce même bulletin (Tchérémissinoff et al., 2005).

L'attribution à un faciès campaniforme de la petite série présentée avait alors fait l'objet de vives critiques, notamment de la part du comité de lecture, en regard du déficit d'éléments typologiques et du profil taphonomique du gisement.
Cependant, les questions sous-tendues par cette réflexion se sont finalement avérées utiles au regard de cette productive table ronde. En effet, la définition de ces faciès «innommables », c'est-à-dire comportant des éléments mixtes situés entre le XXIII ${ }^{\mathrm{e}}$ et le $\mathrm{XX}^{\mathrm{e}}$ siècle avant notre ère, reste compliquée, ne serait-ce que parce qu'ils sont le plus souvent escamotés sous l'appellation générique de Chalcolithique/Bronze ancien, alors qu'ils semblent plutôt déterminants en vue de la qualification même du Bronze ancien I dans de nombreuses régions (cf. conclusion). 
Nous rappellerons donc ici brièvement la démarche réalisée sur le terrain et les différents postulats retenus dans le cadre de l'étude de ce site.

Le contexte d'implantation est celui d'une vaste plaine alluviale, la basse terrasse de la Garonne. Il s'agit essentiellement d'une nappe résiduelle de tessons, fossilisée au sein d'une microdépression. Ces vestiges sont localisés dans la bordure méridionale d'une aire d'environ trois hectares ayant livré différentes occupations se rapportant à la Préhistoire récente, de la fin du Néolithique moyen au Bronze ancien et, ponctuellement, à l'Âge du fer. On observe globalement un gradient chronologique s'étirant d'est en ouest (fig. 1) et des conditions de fossilisation inégales, puisque la terrasse graveleuse sous-jacente présente un profil bosselé irrégulier et que les zones les plus basses ont été scellées plus rapidement.

La nappe qui nous intéresse (fig. 2) s'étend sur $340 \mathrm{~m}^{2}$. Elle a fait l'objet d'une fouille manuelle et tous ses éléments ont été cartographiés. Elle présente des densités inégales et sa conservation s'altère progressivement en direction du nord, ce que nous avons vérifié par le biais de collages poussés sur trois zones (locus A, B et C; Tchérémissinoff et al., 2005). Ainsi, la zone méridionale présente l'image d'une nappe faiblement remaniée avec une forte densité de grands tessons jointifs, alors que la fragmentation augmente au nord lorsque la nappe s'estompe. Puis elle disparaît dans le tiers septentrional, au profit d'un épandage se rapportant au Second Âge du fer, dont la surimpression à la nappe initiale était bien lisible à la fouille.
Le nombre de tessons concernés par l'étude réalisée par Philippe Marsac s'élève à environ 3500 . Ils se rapportent à 27 individus au moins, dont 12 proviennent de la seule zone A. Les éléments typologiques se décomposent en 74 languettes, 9 fonds et 30 bords. La majorité d'entre eux se rapporte à un Chalcolithique ubiquiste, mais certains éléments ont attiré notre attention : la présence de fonds plats, de perforations sousorales, de deux vases à profils sinueux de type gobelet et d'une grande jarre en tonnelet comportant l'association hybride d'une rangée de perforations sous-orales ainsi que de languettes superposées sur sa panse (fig. 3). La restitution de ce vase, qui avait alors essuyé de vives critiques nous paraissant justifiées en termes de représentation graphique (les collages intermédiaires de fragments de panse n'ayant pas été représentés), reste néanmoins très défendable. En effet, les 150 fragments formant le remontage partiel proviennent de la concentration restreinte méridionale (zone $\mathrm{A}^{\mathrm{I}}$ ) et les différents pans possèdent les mêmes caractéristiques techniques. Cela semble d'ailleurs confirmé par l'étude de lames minces réalisée par Fabien Convertini sur les fragments typologiques 1 et 2 (cf. Convertini, ce volume).

Enfin, ce secteur méridional livre aussi plusieurs scies à encoches sur éclats plans en quartzite (étude Marc Jarry) de type chalcolithique (infra).

Dans le cadre de recherches comparatives régionales, nous nous sommes tout d'abord intéressée aux séries voisines. La série céramique du site de Cassagna 1 (Lacombe et al., 2002), étudiée par Marion Viarouge (Viarouge, 2002), a ainsi livré des éléments

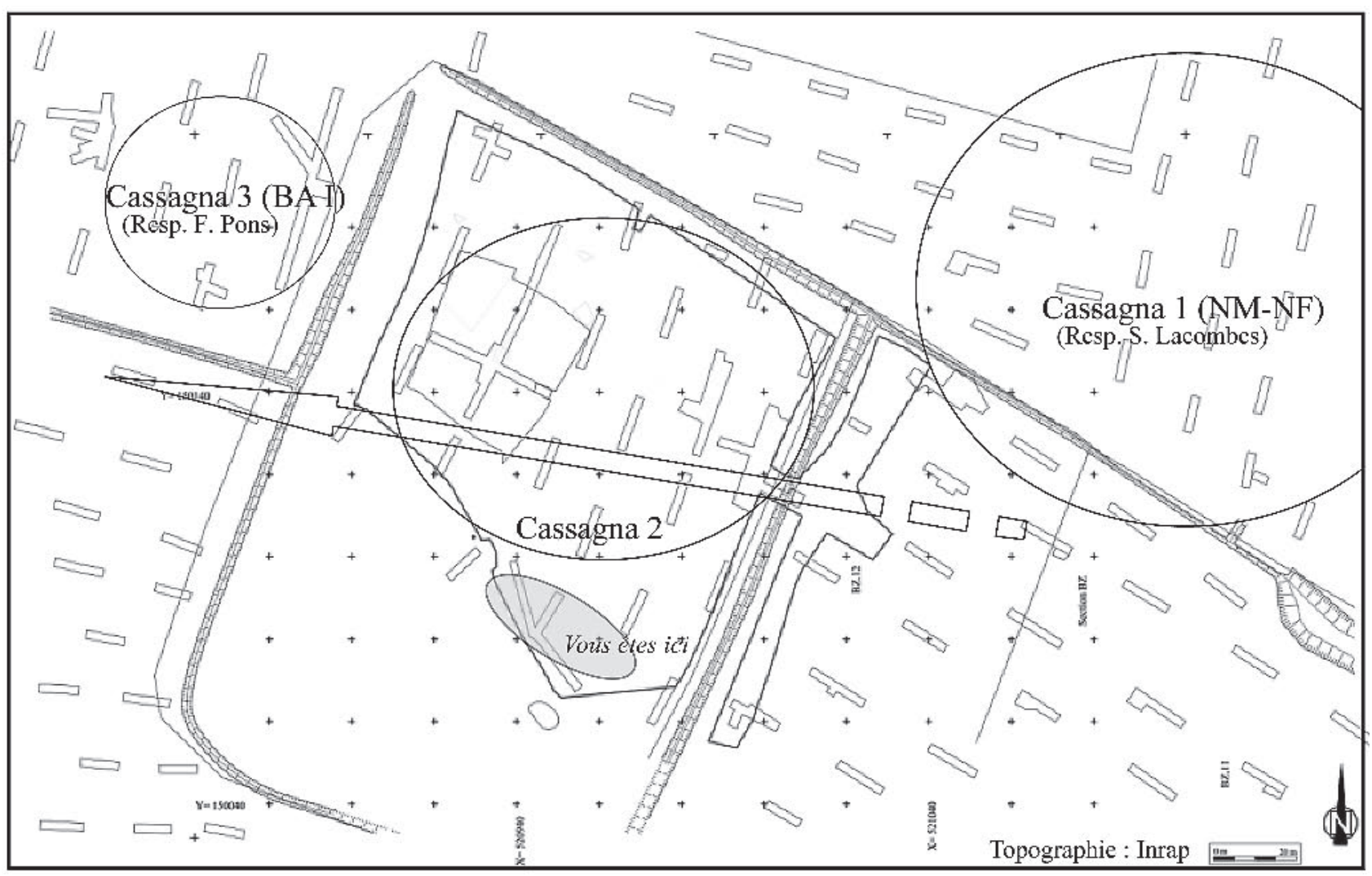

Fig. 1 - Localisation de la surface.

Fig. 1 - Location of the surface. 


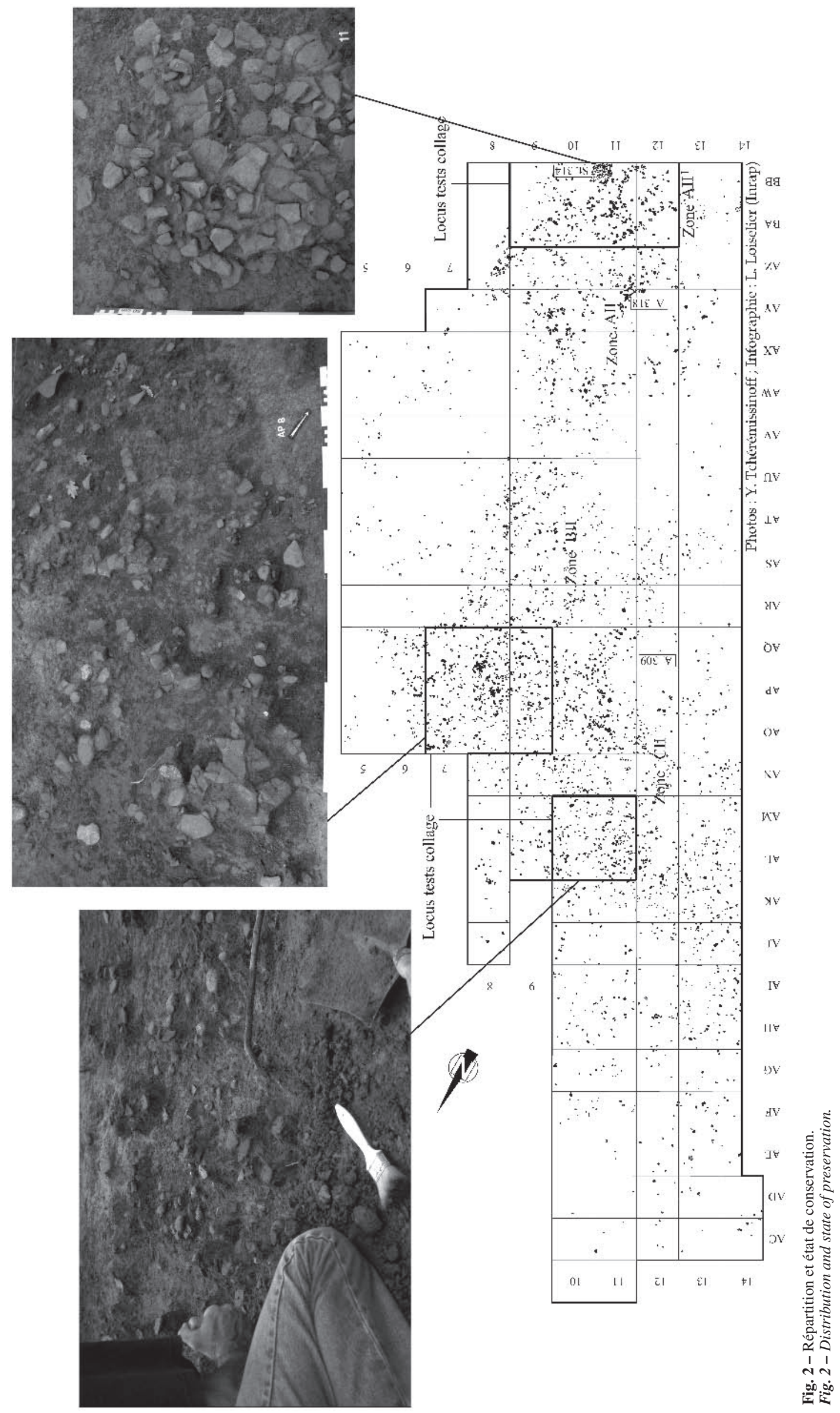




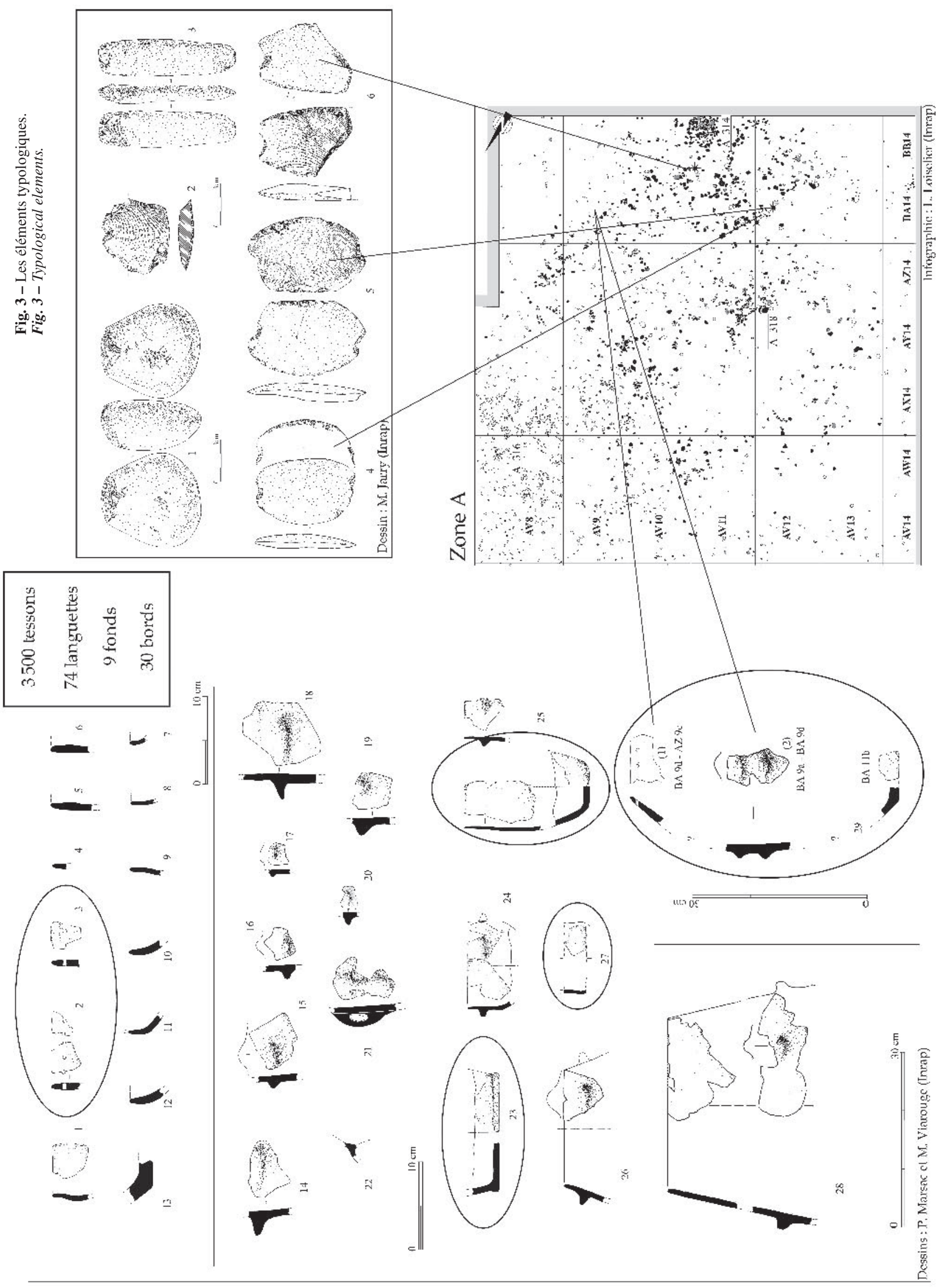




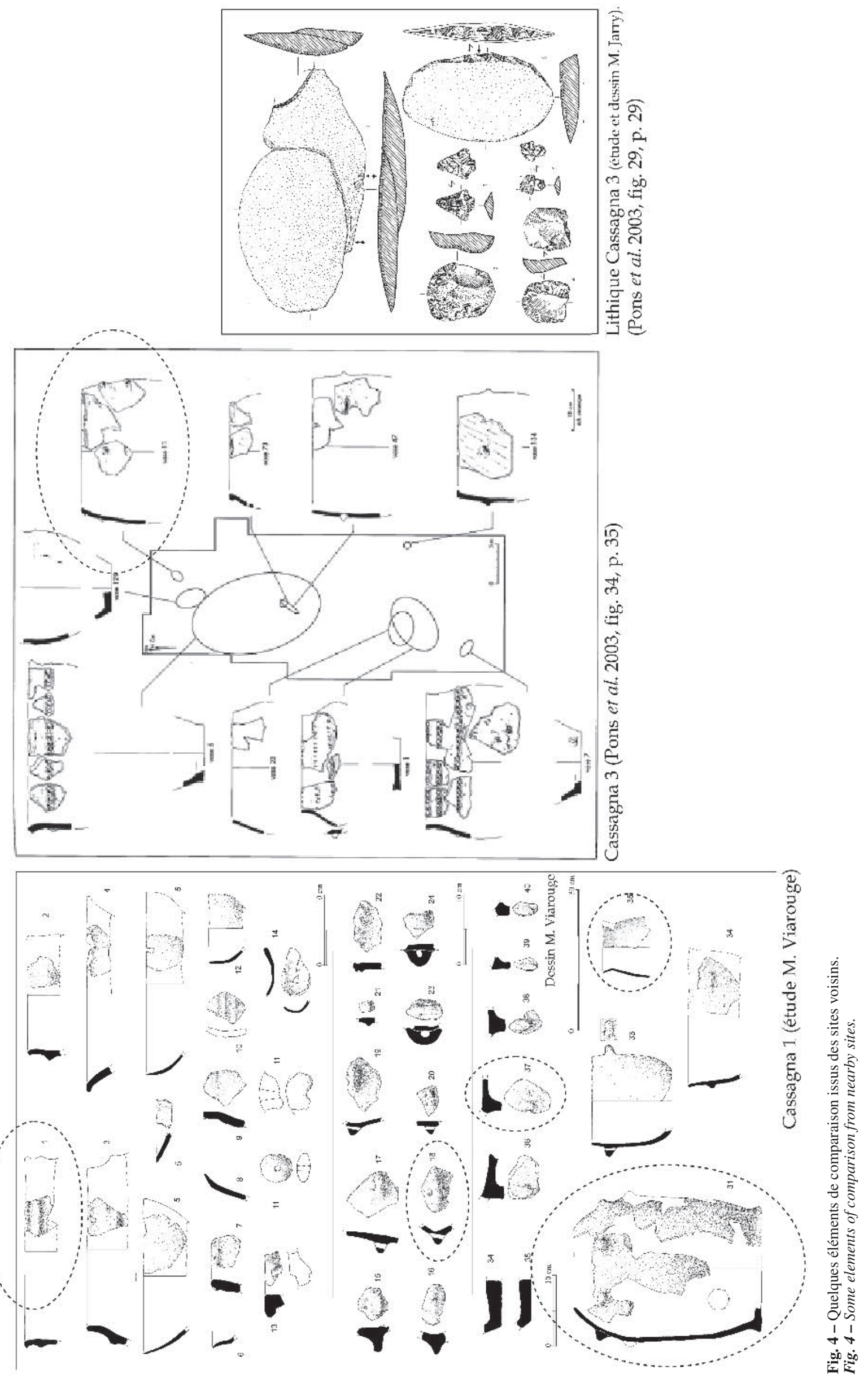




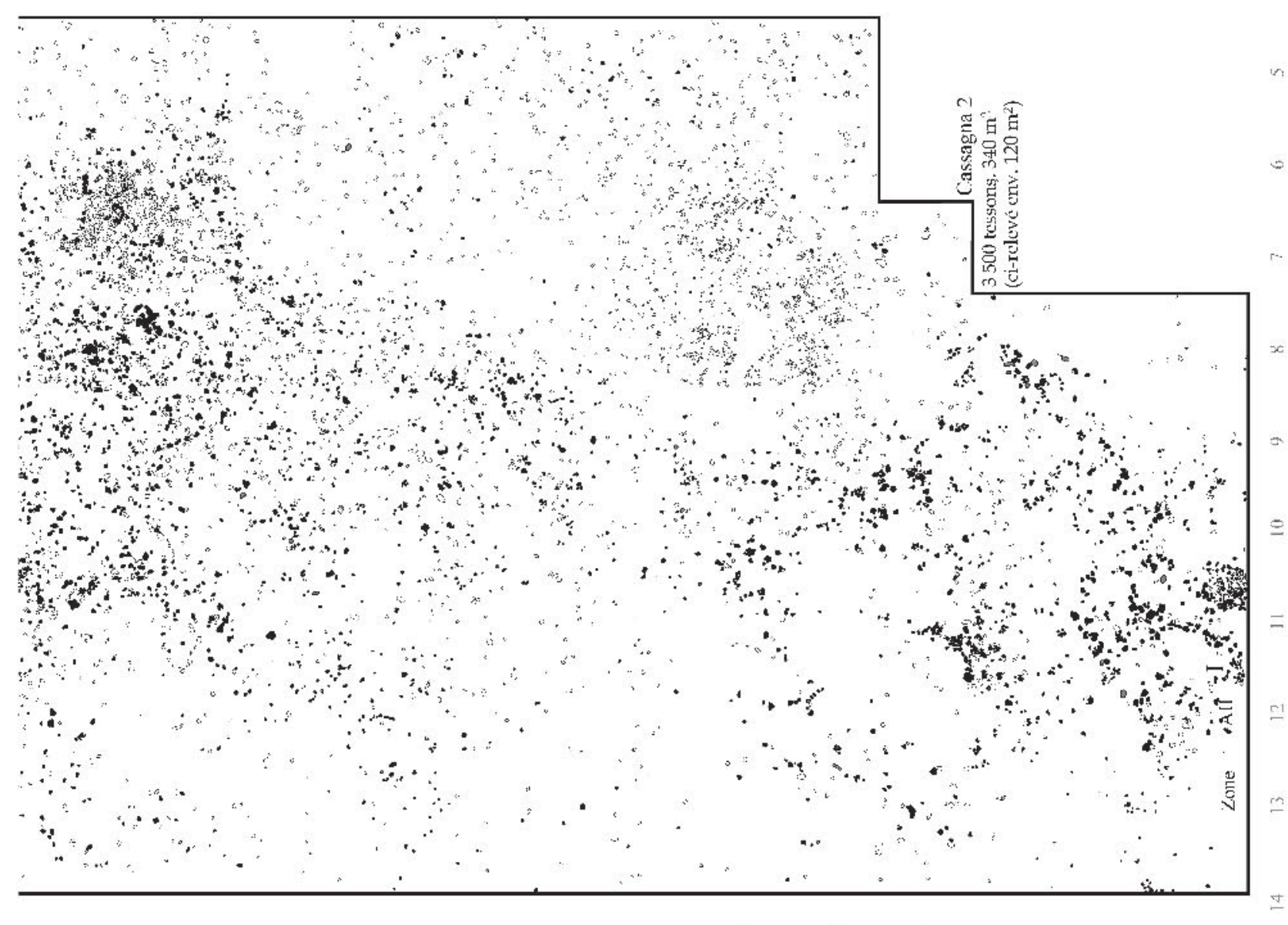

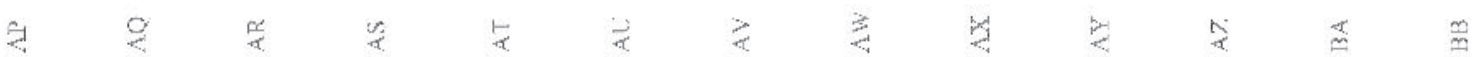

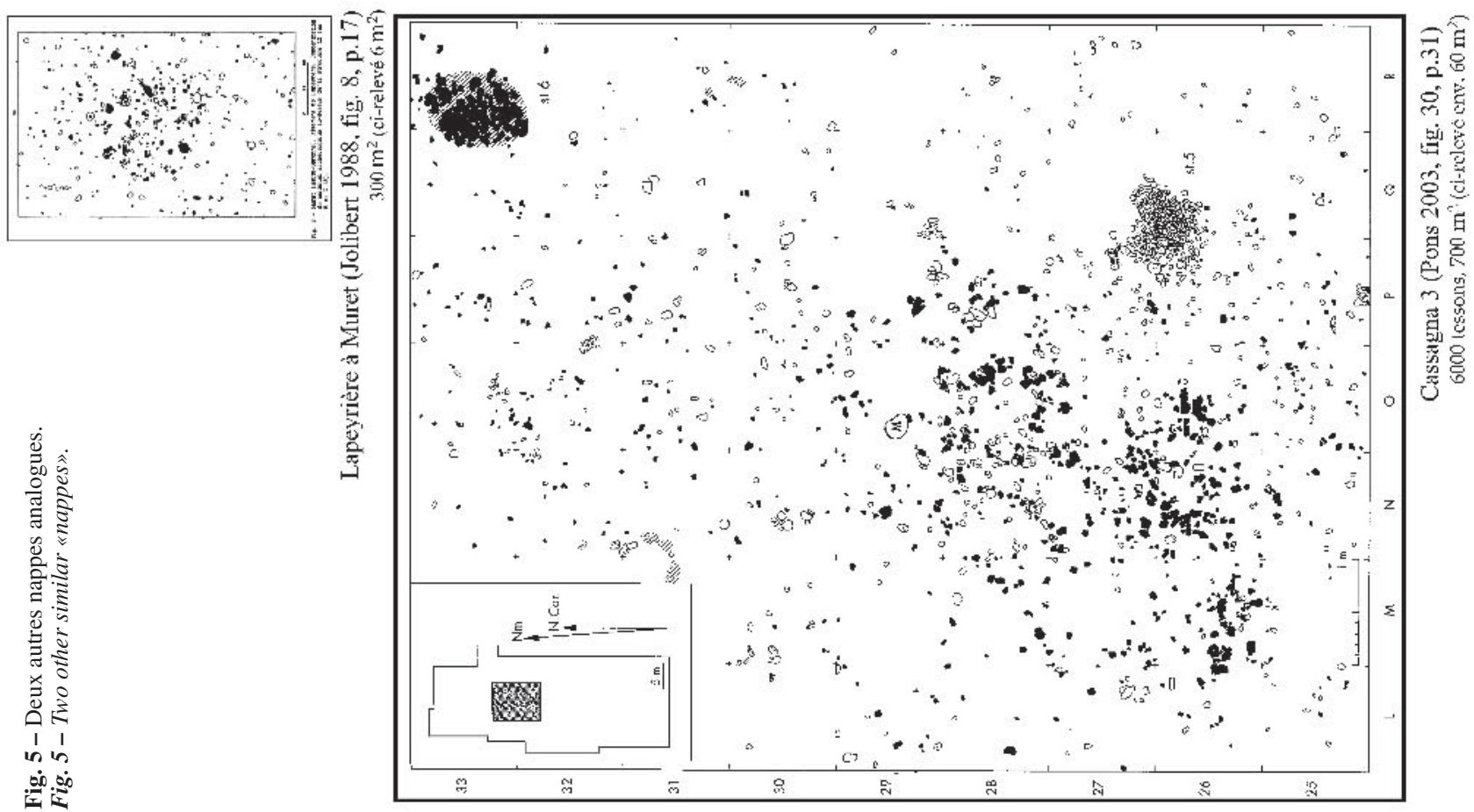


qui nous semblent pertinents pour notre propos (fig. 4), notamment un grand vase en tonnelet portant 6 paires de languettes superposées au niveau de l'épaulement et un fond plat débordant, c'est-à-dire associant incontestablement un caractère vérazien fort à un élément qui, considéré isolément, aurait sans doute été attribué à l'Âge du bronze; les languettes superposées ne constituent donc pas un type exclusif.

La série issue de Cassagna 3 (Pons et al., 2003) se rapporte, elle, explicitement au début du Bronze ancien. Pourtant, la présence de languettes est numériquement très importante et un grand vase porte même au moins deux couples de languettes ou boutons (fig. 4) au niveau de son épaulement, ce caractère demeurant donc possiblement vivace durant le $\mathrm{XX}^{\mathrm{e}}$-XIX $\mathrm{X}^{\mathrm{e}}$ siècle avant notre ère. Concernant la série lithique, également étudiée par Marc Jarry, elle a livré des éclats plans sur quartzite, mais ces couteaux à dos ne sont pas encochés comme c'est le cas sur notre site. Or, ces scies à encoches (plus familièrement qualifiée de navettes) sont considérées comme un bon indicateur du faciès vérazien de la vallée de la Garonne. Nous l'avons donc envisagé comme un argument positif en faveur d'une attribution antérieure à l'Âge du bronze.

Pour l'instant, le seul site garonnais se rapportant explicitement au Campaniforme demeure celui de Lapeyrière à Muret (Jolibert, 1988). Il s'agit également essentiellement d'une nappe de mobilier $\pi^{1}$, dont la densité est comparable à celle de notre site (fig. 5), mais qui a livré plus d'une centaine d'éléments typologiques et plusieurs formes complètes. L'effectif se compose surtout d'une belle série d'éléments se rapportant à l'ensemble méridional de la céramique d'accompagnement (Besse, 2003), de 31 tessons décorés de type pyrénéen, mais aussi de quelques éléments typiquement véraziens. Il n'y a pas d'outils sur éclats plans sur ce gisement.

Dans le cadre de comparaisons plus larges avec des sites campaniformes, on relève aussi que la présence d'éléments décorés n'excède jamais $30 \%$ et que des éléments chalcolithiques demeurent, ces derniers étant généralement considérés comme résiduels.

Concernant la chronologie absolue, les dates sont (logiquement) déficientes pour ces sites. Le site de Cassagna 3 a livré une date qui a peu de chance d'être antérieure au $\mathrm{XX}^{\mathrm{e}}$ siècle avant notre ère (Arc 2145 : $3655 \pm 50$ [2194-1896 BC]) et le site de Muret, une date dont la large fourchette ne peut excéder le XXIII ${ }^{\mathrm{e}}$ siècle (Gif 5970 : $4080 \pm 100$ [2895-2348 BC]).

\section{PROPOSITION}

Dans le cadre de l'étude, la fossilisation de la nappe initiale, surtout lisible dans la portion méridionale, nous a semblé suffisante pour réfléchir sur cette petite série, le principal écueil résidant malheureusement dans son déficit en éléments diagnostiques. Ces derniers présentant cependant une cohérence spatiale, il nous a alors semblé possible de réaliser la proposition suivante :

- les éléments de la série renvoyant à la céramique d'accompagnement du Campaniforme ne constituent pas une pollution, si l'on considère la probable survivance des traits chalcolithiques, vérifiable notamment au sein des séries régionales du Bronze ancien. Il est donc possible que cette série constitue un ensemble homogène que nous avons choisi de qualifier de campaniforme, au regard de la présence de certains caractères et de son antériorité sur la série proche de Cassagna 3 qui renvoie à une phase précoce du Bronze ancien.

Ce postulat sous-tendait les deux questions suivantes :

- la présence d'éléments décorés est-elle indispensable en vue d'une attribution au (à un) Campaniforme?

- faut-il considérer que la présence de languettes, fussent-elles superposées, signe toujours une occupation vérazienne régionalement?

Il paraît évident que la série n'est pas suffisamment étoffée pour pouvoir répondre à ces questions, qui touchent à l'identification d'un faciès local tardif du Campaniforme, ou à celle d'une phase initiale du Bronze ancien. Cependant, nous considérons toujours qu'il était possible de le proposer et nous tenons à remercier ici Laure Salanova à l'initiative de ces débats profitables.

\section{NOTE}

(1) $\left(300 \mathrm{~m}^{2}\right), 16$ de structures à galets et une grande fosse.

\section{RÉFÉRENCES BIBLIOGRAPHIQUES}

BESSE M. (2003) - L'Europe du $3^{e}$ millénaire avant notre ère : les céramiques communes au Campaniforme, Cahiers d'Archéologie romande, 94, Lausanne, 223 p.

JOLIBERT B. (1988) - Le gisement campaniforme de Muret, Archives d'Écologie préhistorique, École des hautes études en Sciences sociales, Toulouse, $135 \mathrm{p}$.

LACOMBE S., DELFOUR G., MARTIN H., VIAROUGE M. (2002) Une occupation du Néolithique final/Chalcolithique : le site de Cassagna 1 à Blagnac (Haute-Garonne), rapport de fouille, INRAP, SRA Midi-Pyrénées, Toulouse, 132 p.

PONS F., LAGARRIGUE A., avec la coll. de COLONGE D., JARRY M., MARTIN H. (2003) - Un habitat de plein air du Bronze ancien dans le Midi toulousain à Blagnac (Haute-Garonne) : le site de Cassagna 3, Documents d'Archéologie méridionale, ${ }^{\circ}{ }^{26}$, p. 7 41.
TCHERÉMISSINOFF Y., MARSAC P., BERTHET A.-L., JARRY M., VIAROUGE M. (2005) - Essai de caractérisation d'un ensemble céramique campaniforme non décoré dans le toulousain : le site de Cassagna 2, Bulletin de la Société préhistorique française, t. 102, $\mathrm{n}^{\circ} 2$, p. $381-400$.

VIAROUGE M. (2002) - Étude de la céramique du site de Cassagna 1 à Blagnac (Haute-Garonne); série du Néolithique final-Chalcolithique, mémoire de maîtrise, université Toulouse-Le Mirail, 1 vol., 177 p.

Yaramila TCHÉRÉMISSINOFF

INRAP-Méditerranée

et CRPPM-UMR 5608-TRACES - Base INRAP

54, avenue du Pont-Juvénal

34000 Montpellier mila.tche@laposte.net 